\title{
Design methodologies for central pattern generators: an application to crawling humanoids
}

\author{
Ludovic Righetti and Auke Jan Ijspeert \\ Biologically Inspired Robotics Group \\ School of Computer and Communication Sciences \\ Ecole Polytechnique Fédérale de Lausanne (EPFL) - Switzerland \\ Email: ludovic.righetti@a3.epfl.ch, auke.ijspeert@epfl.ch
}

\begin{abstract}
Systems of coupled nonlinear oscillators inspired from animal central pattern generators (CPGs) are increasingly used for the control of locomotion in robots, in particular for online trajectory generation. Indeed, such systems present interesting characteristics like limit cycle behavior (i.e. stability), synchronization, and the possibility to be entrained and modulated by external signals. There are now good methodologies for designing systems that exhibit specific gaits, i.e. specific phase relations between oscillators, however techniques to modulate the shape of the rhythmic signals in a controlled way are still missing.

In this article, we present a method for shaping the signals of an oscillatory system according to several criteria that are relevant for locomotion control (but which could also be useful for other applications). These criteria include being able to adjust the relative durations of ascending and descending phases in a cycle, and to temporarily modulate the dynamics of one oscillator according to the states of another one. The first criterion is important for locomotion in order to adjust the duration of swing and stance phases, while the second allows one to introduce signal shape variations to deal with proper inter-limb coordination.

We apply the method to the design of a system of coupled oscillators used to control crawling in a simulated humanoid robot. Using some key characteristics of signal shapes extracted from recordings of baby crawling, we design the system to produce stable trot-like crawling gaits. Insights from symmetry groups' theory are used to design the right phase lags. The oscillators are designed such that the speed of locomotion can be adjusted by varying the duration of the stance phase while keeping the duration of the swing phase constant, like in most tetrapod animals.
\end{abstract}

\section{INTRODUCTION}

This work is part of the RobotCub project, a 5-year European project whose purpose is to build a 54-degrees of freedom humanoid robot with the cognitive abilities of a child [1]. The project has two main goals: first, to create an open and freely-available humanoid platform for research in embodied cognition, and second, to study cognitive development. Like to a child, the robot (called the iCub) should be able to explore its environment by crawling and sitting to manipulate objects.

This contribution presents a design methodology for the crawling controller, based on the Central Pattern Generator (CPG) paradigm. CPGs are neural networks located in the spine of vertebrates and are able to automatically generate the control signals for the coordination of the muscles during periodic movements (e.g. locomotion, respiration) [2], [3]. Although CPGs are controlled by simple descending paths from higher parts of the brain, they are able to generate the signals that control the complex coordination of the muscles during rhythmic movement. CPGs are oscillatory networks that can be modeled as coupled oscillators [4], [5]. Models of CPGs for robotics applications have proven successful, especially for locomotion control where they are used to generate joint trajectories [6]-[8]. Their advantage is that it is easy to modulate the trajectories for locomotion and they have stability properties that makes them suitable for adding feedback pathways. However, very few design methodologies are currently available to construct them [9], [10]. In particular, techniques to modulate the shape of the rhythmic signals in a controlled way are still missing.

Our design methodology follows a biologically inspired approach. Indeed, to design the controller we study the crawling behavior of infants in order to extract important principles for our controller. Then we present a mathematical model of CPG based on coupled nonlinear oscillators to reproduce the crawling gait of infants. The originality of the model resides both in the design of the oscillator and in the design of the coupling scheme of the CPG. Note that while we design a CPG for a specific task, we develop tools that are general enough to be used in other applications.

We designed our oscillator from the observation that the gait pattern of animals and humans can be separated into two distinct phases for each limb. The stance phase is the phase during which the limb touches the ground. The swing phase is the phase during which the limb lifts off the ground. It is a well-known fact that when quadrupeds change their speed of locomotion, they might change their gait and the duration of the stance phase, but the duration of the swing phase tends to remain the same [11]. However, most of the CPG models based on coupled oscillators are not able to separate the swing and stance phases durations. In this contribution, we present an oscillator model in which we can independently control the duration of each of these phases. This is an important feature, since during the swing phase, one limb is off the ground, thus making the system less stable and more dependent on dynamical properties of the controlled robot. So it seems important to control independently the duration of both phases.

Furthermore, we present a coupling scheme based on the analysis of the crawling pattern of real infants to reproduce a similar gait. This coupling is based on the fact that the 


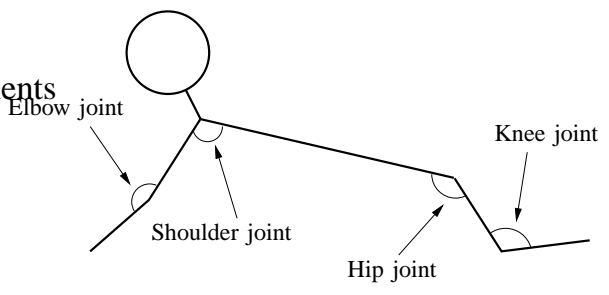

Fig. 1. Schematic of the joint angle we measured. We look at the movement of the limbs in the sagittal plane.

infants have a trot-like gait for the temporal synchronization of the limbs but the stance and swing phases durations are very different compared to most trotting animals. Moreover, it appears that there exists a correlation between the movement of a limb during its stance phase and the swing phase of the opposite limb. We reproduce this influence in the coupling scheme we present and we use the theory of symmetric dynamical systems [12]-[14] to infer the architecture of the network of coupled oscillators. The validation of the design is done by testing the CPG controller with a physics simulation of the $\mathrm{iCub}$, since the real robot is still under construction.

In the next section, we first review data on crawling in infants (Section II). We then present the design approach behind our model of coupled oscillators (Section III). The design is done incrementally with first the construction of a nonlinear oscillator with two controlled time scales, then the addition of inter-limb influences between oscillators of opposite limbs, and finally the addition of inter-limb couplings between the complete four-oscillator system for implementing the trot-like crawling gait. The model is tested with a rigid articulated body simulation of the iCub, and compared to the original infant crawling gaits (Section IV). The paper concludes with a short discussion (Section V).

\section{CRAWLING IN INFANTS}

Very few studies about crawling in babies have been made. Some psychological studies about the development of crawling in infants are available [15], [16] but they all focus on the cognitive development of infants through locomotion and none have focused on the kinematic details of crawling babies.

Babies can have various types of crawling and in this contribution we only focused on the standard gait [15]. Indeed, this gait is the most widespread one among infants and for a first study of crawling it seemed to be the most appropriate. This gait corresponds to the locomotion of the baby on its hands and knees, with the phase relation of a trot. More information about the different types of crawling can be found in [15].

In order to study crawling in infants, we used recordings of the trajectories of the limbs of crawling babies. These recordings were made in the Department of Psychology at Uppsala University, with a Qualisys Motion Capture System. These recordings where then converted into joint angle coordinates because this is a more natural coordinate frame for the control of a robot. Figure 1 shows the basis for the joint

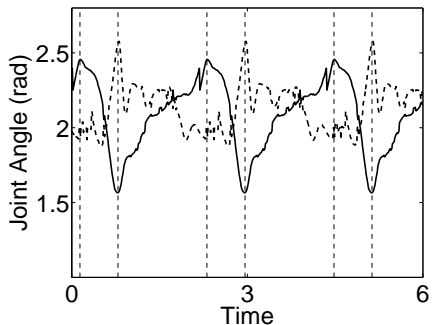

(a) Left Arm

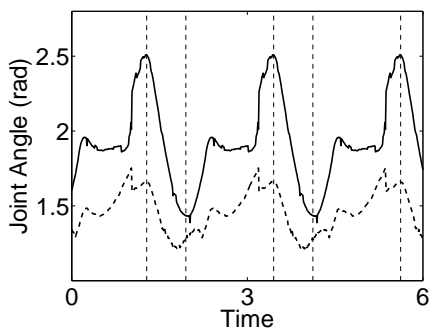

(c) Left Leg

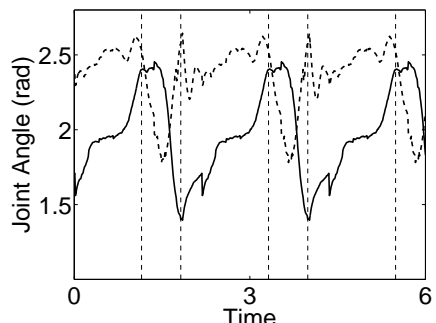

(b) Right Arm

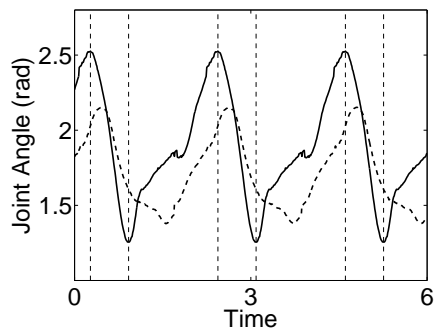

(d) Right Leg
Fig. 2. Typical evolution of the joint angles of a baby during crawling. This is a reconstruction of a crawling sequence from the recordings of a crawling baby. We plot the joint angles (in radian) of the 4 limbs. For each limb, we plot the joint angles as defi ned in Figure 1. Hip and shoulder joints are plotted in plain line, the knee and elbow joints are in dashed line. The vertical lines delimit the swing and stance phases, the swing phase being the shortest one.

angles and Figure 2 shows a typical crawling gait we got from the recordings. In this study we only focus on the joint angles in the sagittal plane.

The first general remark we can state from these data is that standard crawling is a trot-like gait for its temporal relations between limbs. It means that the diagonal limbs (e.g. left arm and right leg) are in phase and half a period out of phase with the opposite limbs. However, this trot-like gait is very different from trot gaits found in animals. Generally, the trot gait is a medium speed gait between walk and gallop and the duration of the stance phase is quite short compared to the swing phase. The baby crawling gait has different properties in terms of these relative durations. Indeed, the stance phase is really long compared to the swing phase, it represents about $70 \%$ of a whole cycle. A comparative study between the kinematics of crawling babies and monkeys can be found in [17] and supports these remarks about the crawling gait and the relative durations of the swing and stance phases.

When looking at the movement of the hip and shoulder joints (as defined in Figure 1), we notice that during the stance phase the joint slows down or even sometimes stops during the swing phase of the opposite limb. It is as if the swing phase of a limb was inhibiting the movement of the opposite limb. This observation is also supported by the data shown in [17], although this fact is not mentioned by the authors.

The knee joint of each limb is folding in order to follow 
the movement of the hip. Since the baby is crawling on its knees, the exact control of this joint is less important (i.e. the tibia tends to simply rest on the ground). The elbow joints are folding during the swing phase, to allow the arm to reach a further region in front of the baby but do not move significantly during the stance phase.

Our goal here is not to study in detail the crawling sequence of the baby, but to extract the features that seem important in order to reproduce the same gait in a robot. The main features we would like to emphasize from these observations and from the study of [17] are first that the crawling gait is a trot-like gait in terms of phase relations between the limbs but with a stance phase that is much longer than the swing phase contrary to usual trot gaits. Second, there is a correlation between the swing phase of a limb and the arrest of movement of the hip (or shoulder) joint of the opposite limb. Third, the elbow is folding to allow the arm to do the swing phase.

\section{MODEL}

In this section we construct a model of CPG by means of coupled oscillators. The CPG will be used to generate the crawling trajectories for the iCub humanoid robot. To construct a CPG model, we define a number of features we would like our model to have.

From biology, we know that during locomotion at various speeds, the duration of the swing phase of animals is always constant. Only the duration of the stance phase (and the change of gait) influence the speed of locomotion [3], [11]. Thus, we would like to be able to control independently the duration of each of these phases in the CPG. From the observations of the previous section, we would like our CPG to generate a trot-like gait, with inhibition of the movement of the hip and shoulder joints during the swing phase of the opposite limbs.

From a robotics point of view, the CPG must have properties that makes it suitable for the control of a real robot. We therefore want the CPG to show limit cycle behavior and to be stable against perturbations, to allow for further integration of sensory feedback. We also want to be able to smoothly modulate the generated trajectory in frequency and in amplitude to have a larger range of possible locomotion.

In summary our CPG must have the following properties

- Smooth modulation of the generated trajectory in frequency and amplitude

- Independent control of the duration of the swing and stance phases (the ascending and descending phases)

- Trot-like gait with a stance phase much longer than the swing phase

- Inhibition of the movement of the hip and shoulder joints during the swing phase of the opposite limbs

- Stability to perturbations to allow feedback integration

\section{A. Two time-scale oscillator}

We first present a model of a stable oscillator with the possibility to control independently the duration of the swing and stance phases and the amplitude of the oscillations.
If we take a simple spring-like oscillatory system, the equation of motion of the joint angle can be expressed as

$$
\begin{aligned}
& \dot{x}=y \\
& \dot{y}=-K x
\end{aligned}
$$

The frequency of oscillations will be $\sqrt{K}$ and we will have harmonic oscillations whose amplitude will depend on the initial conditions of the system.

We want a duration of the stance phase different from the duration of the swing phase, thus we can think of an oscillator changing its spring constant according to the phase. It will have a $k_{\text {stance }}$ spring constant during stance phase and $k_{\text {spring }}$ constant during swing phase. The oscillator will switch among these two constants according to the phase, that is according to the sign of the velocity $y$ of the system. We can thus write a general spring constant as

$$
K=k_{\text {stance }}+\left(k_{\text {swing }}-k_{\text {stance }}\right) \frac{1}{\mathrm{e}^{\text {by }}+1}
$$

where the exponential function works as a step function which selects either $k_{\text {swing }}$ or $k_{\text {stance }}$ according to the sign of the velocity of movement $y, b$ controls the speed of the switch.

Now we have a system that oscillates with different speeds according to the direction of the oscillations. Therefore we can independently control the duration of the swing and the stance phases.

The problem with such an oscillatory system is that no limit cycle exists. There exist infinitely many periodic orbits around the unstable center 0 and thus the system is not stable. We can point the flow toward one periodic orbit by constraining the total energy of the system, since it defines the maximum value $x$ can take in a spring system. The total energy of the system is defined by

$$
E=\frac{1}{2}\left(K x^{2}+y^{2}\right)
$$

which is the sum of the potential and kinetic energies of the system (we take the mass equal to one). At $y=0$ we have $E=\frac{1}{2} K x^{2}$, which gives $x_{\max }= \pm \sqrt{\frac{2 E}{K}}$. We can choose a total energy such that $x_{\max }$ is bounded to a certain value, $E=\frac{\mu^{2} K}{2}$ and $x_{\max }= \pm \mu$.

In order to constraint the amplitude of oscillations, we add a damping term to the preceding equation which bounds the total energy of the system. We then rewrite the whole system as

$$
\begin{aligned}
& \dot{x}=y \\
& \dot{y}=\alpha y\left(\mu^{2} K-\left(K x^{2}+y^{2}\right)\right)-K x
\end{aligned}
$$

where $\alpha$ is a constant controlling the speed of convergence of the energy of the system $\frac{1}{2}\left(K x^{2}+y^{2}\right)$ to the wanted total energy $\frac{1}{2} \mu^{2} K$.

The stability of the system can be seen if we set $E=$ $\frac{1}{2}\left(K x^{2}+y^{2}\right)$, then differentiation with respect to time gives

$$
\dot{E}=\frac{1}{2} \dot{K} x^{2}+\alpha y^{2}\left(K \mu^{2}-E\right)
$$



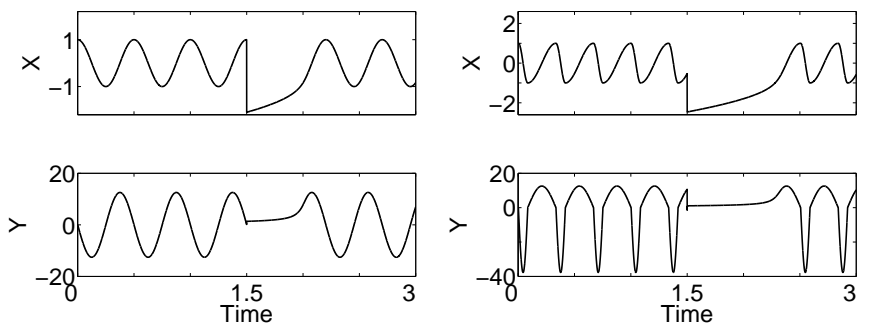

(a) $k_{\text {stance }}=k_{\text {swing }}$

(b) $k_{\text {stance }}=\frac{1}{3} k_{\text {swing }}$

Fig. 3. This fi gure shows how we can independently control the ascending and descending durations of the oscillator, in 3(a) we plot the oscillations when $k_{\text {stance }}=k_{\text {swing }}=4 .(2 \pi)^{2}$, in $3(\mathrm{~b})$ we plot $k_{\text {stance }}=\frac{1}{3} k_{\text {swing }}=$ 4. $(2 \pi)^{2}$. In each plot we show the oscillations $x$ and the velocity $y$. At time $t=1.5$, we perturb the system by setting $x$ and $y$ to a random value, we clearly see that the oscillations are stable.

In our application, $K$ can be approximated as a switching function, whose value equals either $k_{\text {swing }}$ when $y<0$ or $k_{\text {stance }}$ when $y>0$. Thus, for $y \neq 0$, we see that the flow is always directed toward $E=K \mu^{2}$. When $y=0, K$ changes its value from a spring constant to the other and we see that $E$ changes also its value in the direction of this change because of the $\dot{K}$ term. Thus the flow is always directed toward $K \mu^{2}$ and the system is stable. The stable limit cycle has then equation $K x^{2}+y^{2}=K \mu^{2}$. It is composed of two half-ellipses that share the same semi-minor axis $\mu$ (so they are connected) and with foci at $y= \pm \mu \sqrt{k_{\text {swing }}-1}$ and $y= \pm \mu \sqrt{k_{\text {stance }}-1}$ respectively.

Now we have an oscillator bounded in energy for which we can independently control the duration of the swing and the stance phases. Moreover, with the bounded energy, we assure that the oscillator is stable and that we can control the amplitude of the oscillations which are equal to $\mu$. Figure 3 shows an example of oscillations with different values for the stance and the swing phases.

\section{B. Inhibitory coupling}

In this section we describe the inhibitory coupling scheme we use to replicate the slow down of the hip and shoulder joints during the swing phase of the opposite limbs. We introduce an inhibitory coupling that sets the stance spring constant $k_{\text {stance }}$ to 0 when the opposite limb starts its swing phase, i.e. when the speed of the oscillator becomes negative

$$
K_{i}=\frac{k_{\text {stance }}}{\left(\mathrm{e}^{-b y_{i}}+1\right)\left(\mathrm{e}^{-k y_{j}}+1\right)}+\frac{k_{\text {swing }}}{\mathrm{e}^{b y_{i}}+1}
$$

where $i$ denotes the oscillator that is inhibited and $j$ the opposite oscillator, $k$ controls the speed of slow down of the oscillator. With this coupling scheme, when one limb starts its swing phase, the opposite limb will slow down its movement. The amount of deceleration will depend on the kinetic energy the oscillator has at this moment and the energy damping term $\alpha$.

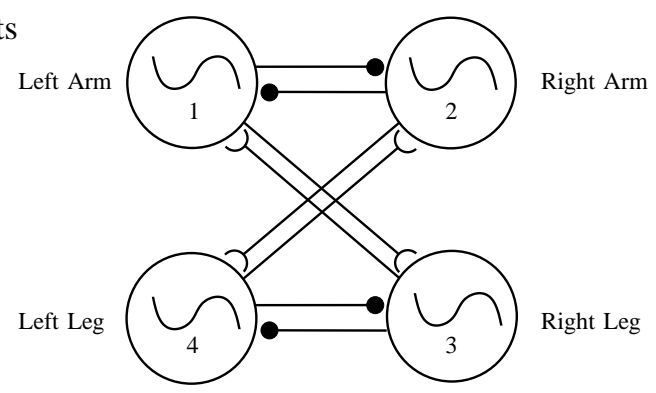

Fig. 4. The architecture of the CPG

\begin{tabular}{|c|c|c|}
\hline $\mathrm{K}$ & Possible Periodic Solutions & Stability \\
\hline$\Gamma$ & $x_{1}(t)=x_{2}(t)=x_{3}(t)=x_{4}(t)$ & Unstable \\
$\{I,(12)(34)\}$ & $x_{1}(t)=x_{2}(t)=x_{3}\left(t+\frac{T}{2}\right)=x_{4}\left(t+\frac{T}{2}\right)$ & Unstable \\
$\{I,(13)(24)\}$ & $x_{1}(t)=x_{2}\left(t+\frac{T}{2}\right)=x_{3}(t)=x_{4}\left(t+\frac{T}{2}\right)$ & Asym. stable \\
$\{I,(14)(23)\}$ & $x_{1}(t)=x_{2}\left(t+\frac{T}{2}\right)=x_{3}\left(t+\frac{T}{2}\right)=x_{4}(t)$ & Unstable \\
\hline
\end{tabular}

Fig. 5. From the symmetry of the network, we derived the possible pattern of synchronization according to the possible subgroups of spatial symmetry. For each subgroup, we indicate the possible periodic solutions and their stability. The stability of the solutions was evaluated numerically, as shown in Figure 6.

To assure that this slow down will be fast enough when $x \simeq 0$, we change the damping term so it has a very high value when $x \simeq 0$ and a smaller value otherwise. To do this, we transform $\alpha$ into a Gaussian function centered around 0 .

$$
\alpha_{i}=\nu\left(1+\beta \mathrm{e}^{-\sigma x_{i}^{2}}\right)
$$

where $\nu$ is the damping constant, $\beta$ controls the change of the damping around 0 and $\sigma$ controls the width of the Gaussian. With $\alpha_{i}$ we can now independently control the general damping term that constrains the total energy of the system and the damping when $x \simeq 0$, i.e. during the inhibition.

\section{Architecture of the CPG}

In addition to the coupling scheme for inhibition, we have to introduce a coupling to maintain the phase relations between each limb. We want a half a period out of phase relation between opposite limbs (e.g. between the arms) and an inphase relation between diagonal limbs (e.g. right arm and left leg).

To design such a network, we use the theory of symmetric coupled cell networks [12]-[14]. By looking only at the symmetries of a network of coupled oscillators, we can deduce the existence of stable solutions having the same symmetries. These symmetries are defined as the group of permutations of the cells of the network which preserve its architecture.

Of course, the symmetries of the network induce that the corresponding ordinary differential equations (ODEs) describing the network have the same symmetry. In this case we can distinguish two kinds of symmetries. The spatial symmetries of a certain set of ODEs which are the symmetries $\gamma$ such that for any solution $x(t)$ of the set of ODEs $\gamma x(t)=x(t)$. The spatio-temporal symmetries are the symmetries $\varphi$ which preserve the orbit of a solution, which means that if $x(t)$ is a solution with orbit $\{x(t)\}$, then $\varphi x(t)$ has the same orbit. In 


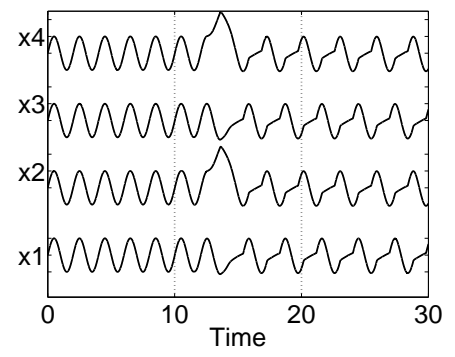

(a) $\Gamma$ symmetry

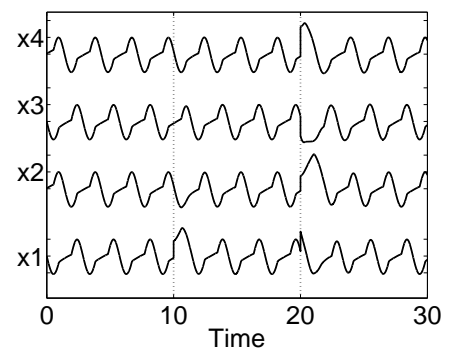

(c) $\{I,(13)(24)\}$ symmetry

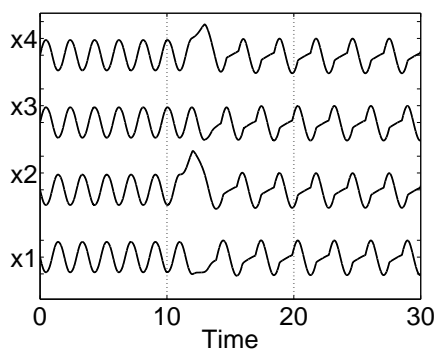

(b) $\{I,(12)(34)\}$ symmetry

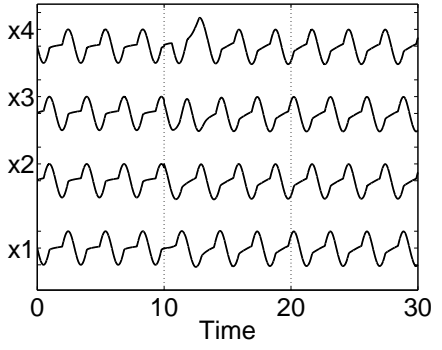

(d) $\{I,(14)(23)\}$ symmetry
Fig. 6. We show the 4 possible patterns of synchrony we predicted from the symmetries of the network. We also show their stability properties by perturbing the oscillators. For patterns of Figs. 6(a) and 6(b) at time $t=10 \mathrm{~s}$ we add a perturbation of 0.01 to $x_{1}$, we see that such a small perturbation completely destroys the patterns and the crawling pattern appears. For the pattern of Figure $6(\mathrm{c})$, which is the crawling pattern, at time $t=10$ we add 1.0 to $x_{1}$ and at time $t=20$ we set the state variables of each oscillator at a random value between $[-2,2]$, it is clear that this pattern is stable. The pattern in Figure 6(d) is a pace gait, at time $t=10 \mathrm{~s}$ we add a random noise between $[-0.2,0.2]$ on each $x_{i}$. For all the experiment, we set $k_{\text {swing }}=$ $k_{\text {stance }}=\pi^{2}, c 1=c 2=1.0, \beta=100, \sigma=10, \nu=0.45, b=k=100$ and $\mu=1$.

other word, if $x(t)$ is a periodic solution, then $\varphi x(t)$ will be the same solution with some phase shift.

For the crawling gait, if we number the limbs as in Figure 4 , we want the permutation of the diagonal limbs $(13)(24)$ to be a spatial symmetry and $\left((12)(34), \frac{1}{2}\right)$ and $\left((14)(23), \frac{1}{2}\right)$ to be spatio-temporal symmetries with half a period phase shift.

We can construct a coupled cell network that is symmetric under the group generated by these symmetries. By the $\mathrm{H} / \mathrm{K}$ theorem, we know that the crawling gait is a periodic solution of any network having the same symmetries.

Theorem 1: $H / K$ Theorem [14] Let $\Gamma$ be the symmetry group of a coupled cell network in which all cells are coupled and the internal dynamics of each cell is at least twodimensional. Let $K \subset H \subset \Gamma$ be a pair of subgroups. Then there exist periodic solutions to some coupled cell systems with spatio-temporal symmetries $H$ and spatial symmetries $K$ if and only if $H / K$ is cyclic and $K$ is an isotropy subgroup. Moreover, the system can be chosen so that the periodic solution is asymptotically stable.
In our case, we have

$$
\Gamma=H=\left\{I,((13)(24), 0),\left((12)(34), \frac{1}{2}\right),\left((14)(23), \frac{1}{2}\right)\right\}
$$

and

$$
K=\{I,((13)(24), 0)\}
$$

We clearly see that $H / K \cong \mathbb{Z}_{2}$ is cyclic and thus the trot-like gait exists as a solution of the system as long as we choose a coupling scheme such that $K$ is an isotropy subgroup (which is easy).

We just have to choose a coupling such that the trot gait is stable, but we already know that it is possible. Since we have inhibitory coupling between opposite limbs, we add standard subtractive coupling between these oscillators in order to enforce the half a period phase shift. As we also want in-phase relations between diagonal limbs, we also add additive coupling between opposite limbs. These couplings are well studied and we know that they make the desired phase shifts between 2 oscillators stable [18]. Figure 4 shows the architecture of the network with the coupling scheme.

The general equations of the CPG that generates the trajectories for the hip and shoulder joints are then

$$
\begin{aligned}
\dot{x}_{i} & =y_{i} \\
\dot{y}_{i} & =\alpha_{i} y_{i}\left(K_{i}\left(\mu^{2}-x_{i}^{2}\right)-y_{i}^{2}\right)-K_{i} x_{i}-c_{1} y_{j}+c_{2} y_{k} \\
K_{i} & =\frac{k_{\text {stance }}}{\left(\mathrm{e}^{-b y_{i}}+1\right)\left(\mathrm{e}^{-k y_{j}}+1\right)}+\frac{k_{\text {swing }}}{\mathrm{e}^{\text {byi }}+1} \\
\alpha_{i} & =\nu\left(1+\beta \mathrm{e}^{-\sigma x_{i}^{2}}\right)
\end{aligned}
$$

where $i=1 \ldots 4$ denotes the ith oscillator, $j$ the opposite oscillator and $k$ the diagonal oscillator, $c_{1}$ and $c_{2}$ are positive coupling constants. We can verify that $K$ is an isotropy subgroup for this set of equations.

Another advantage of this method to design the architecture of the CPG is that we can directly calculate the existence of other patterns of oscillations by simply calculating the other subgroups of $\Gamma$ as is shown in Figure 5. It is very important to be able to calculate the possible patterns of oscillations and to investigate their stability properties in order to be able to guarantee the behavior of our controller when adding feedback loops. This method transforms the analytic problems of finding these modes of oscillations into an algebraic one, which is easier.

We see that there exist three other oscillatory regimes and we evaluated numerically the stability of each of these patterns, as can be seen in Figure 6. We note that the only stable pattern of oscillation with a wide basin of attraction is the trot gait. The pace gait has a small region of stability that is limited and for a random noise between $[-0.2,0.2]$, this pattern disappears. The two other patterns are unstable. After the perturbations, all these patterns converge to the trot gait.

Now we have a CPG that can generate the trajectories for the hip and shoulder angles. This CPG is stable against perturbations. We can also smoothly modulate the frequency of the pattern by changing independently the frequency of the 


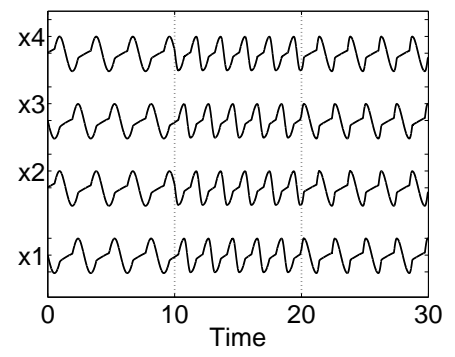

(a) Modulation of the frequency

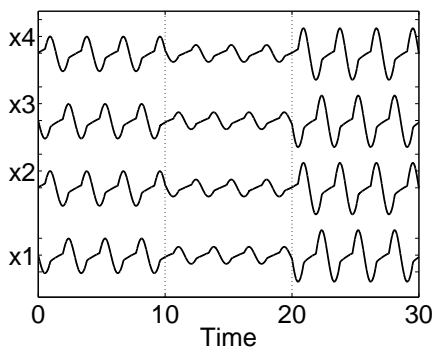

(b) Modulation of the amplitude
Fig. 7. In Figure 7(a) we modulate the frequency of the CPG. Initially we have $k_{\text {swing }}=k_{\text {stance }}=\pi^{2}$, at $t=10$ we set $k_{\text {swing }}=4 k_{\text {stance }}=$ $4 \pi^{2}$, which corresponds to a doubling of the speed of the swing and at $t=20$ we set $k_{\text {stance }}=4 k_{\text {swing }}=4 \pi^{2}$. In Figure $7(\mathrm{~b})$ we modulate the amplitude of the pattern, we set $\mu=1$ at $t=0$, then $\mu=0.5$ at $t=10$ and $\mu=1.5$ at $t=20$. Note that an abrupt change in the control parameters $\left(k_{i}, \mu\right)$ leads to a smooth transition in the generated pattern.
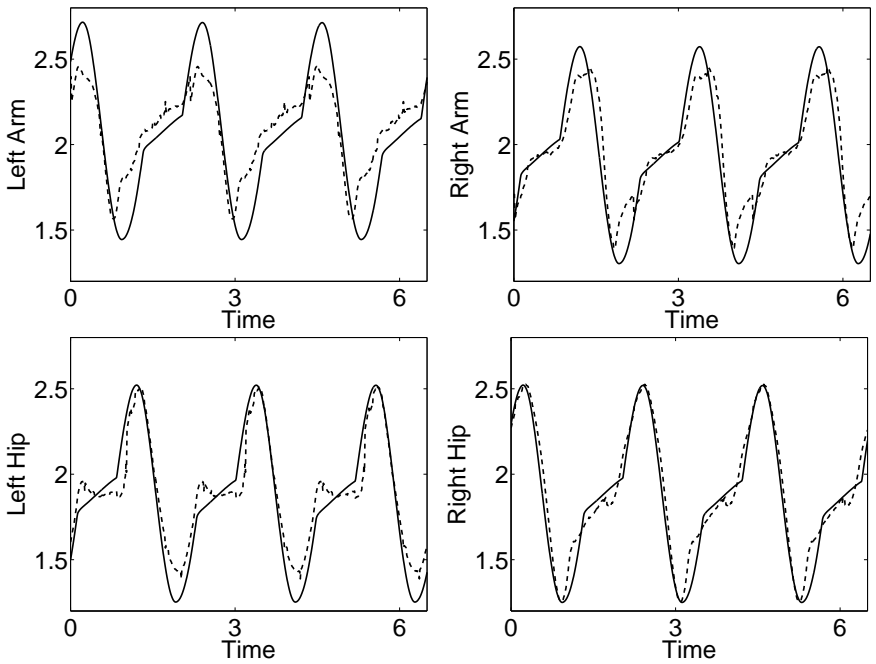

Fig. 8. Comparison of the real trajectories of the hip and shoulder joints and the trajectories generated by the CPG. The trajectories of the CPG are only shifted to oscillate around the same mean values as the real trajectories. We see that the trajectories generated by our model fit quite well the real ones, especially for the right limbs.

ascending and descending oscillations. A smooth modulation of the amplitude is straightforward by changing the parameter $\mu$. Examples of such modulations can be seen in Figure 7.

\section{VALIDATION OF THE MODEL}

\section{A. Comparison with the real baby}

In this section we compare the trajectories of the shoulder and hip joints of a baby with the ones generated by the CPG. In Figure 8 we see the result of the comparison. The theoretical trajectories match quite well the experimental ones. This result shows that the CPG can reproduce the main features of crawling and therefore it supports our design methodology.

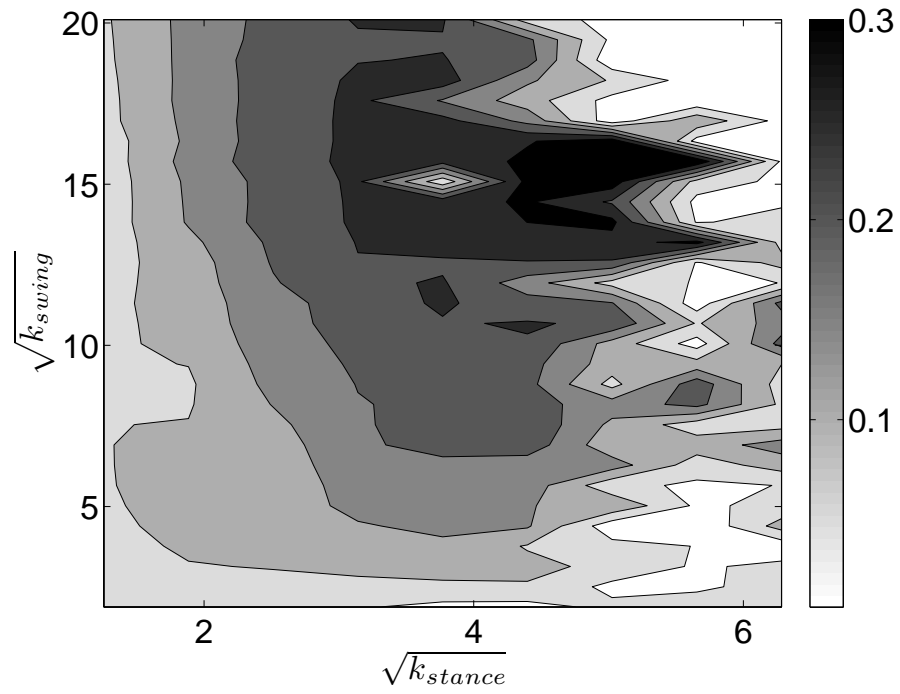

Fig. 9. Characterization of the speed of the simulated iCub according to the duration of the stance and swing phases. The left color bar shows the correspondence between the colors and the speed (in $\mathrm{m} \cdot \mathrm{s}^{-1}$ ).

\section{B. Crawling on the simulated robot}

In this section we show experiments where we use our CPG to control a crawling simulated robot. The simulation is done with Webots [19], a simulator based on ODE [20], an open source physics engine for simulating 3D rigid body dynamics. The simulation is as close as possible to the robot currently under construction. This means that we use the correct lengths and mass distributions for each limbs.

The CPG we developed generates the trajectories for the hip and shoulder joints, so we use these trajectories to control the position of the hip and shoulder joints. However, we saw in Section II that the elbow was also used during the swing phase of the corresponding arm. The elbow is folding during the swing phase, allowing the arm to reach the region in front of the baby.

We thus set the angle of the elbow joint according to the phase of the arm, that is, according to the sign of $y$. The angle of the elbow, $\theta_{i}$, will follow a Gaussian movement corresponding to

$$
\theta_{i}=\gamma \mathrm{e}^{-\frac{\left(y_{i}-\sqrt{k_{\text {swing }}}\right)^{2}}{\tau}}
$$

where $i$ corresponds to the left or right arm, $\gamma$ is the amplitude of the movement and $\tau$ is the width of the Gaussian. The Gaussian is centered on $-\sqrt{k_{\text {swing }}}$ which corresponds to the maximum speed of the shoulder during the swing phase. The oscillator reaches this value at $x \simeq 0$. The elbow will then fold during the swing phase, following a Gaussian movement and will not move during the stance phase. We also control the DOF of the arm, which is orthogonal to the sagittal plane, in the same way. This allows the hand to have more height during the swing phase. Figure 10 shows a crawling sequence of the simulated robot and of a real baby.

We also investigated the importance of the $k_{\text {swing }}$ and $k_{\text {stance }}$ constants for the speed of locomotion of the robot. 

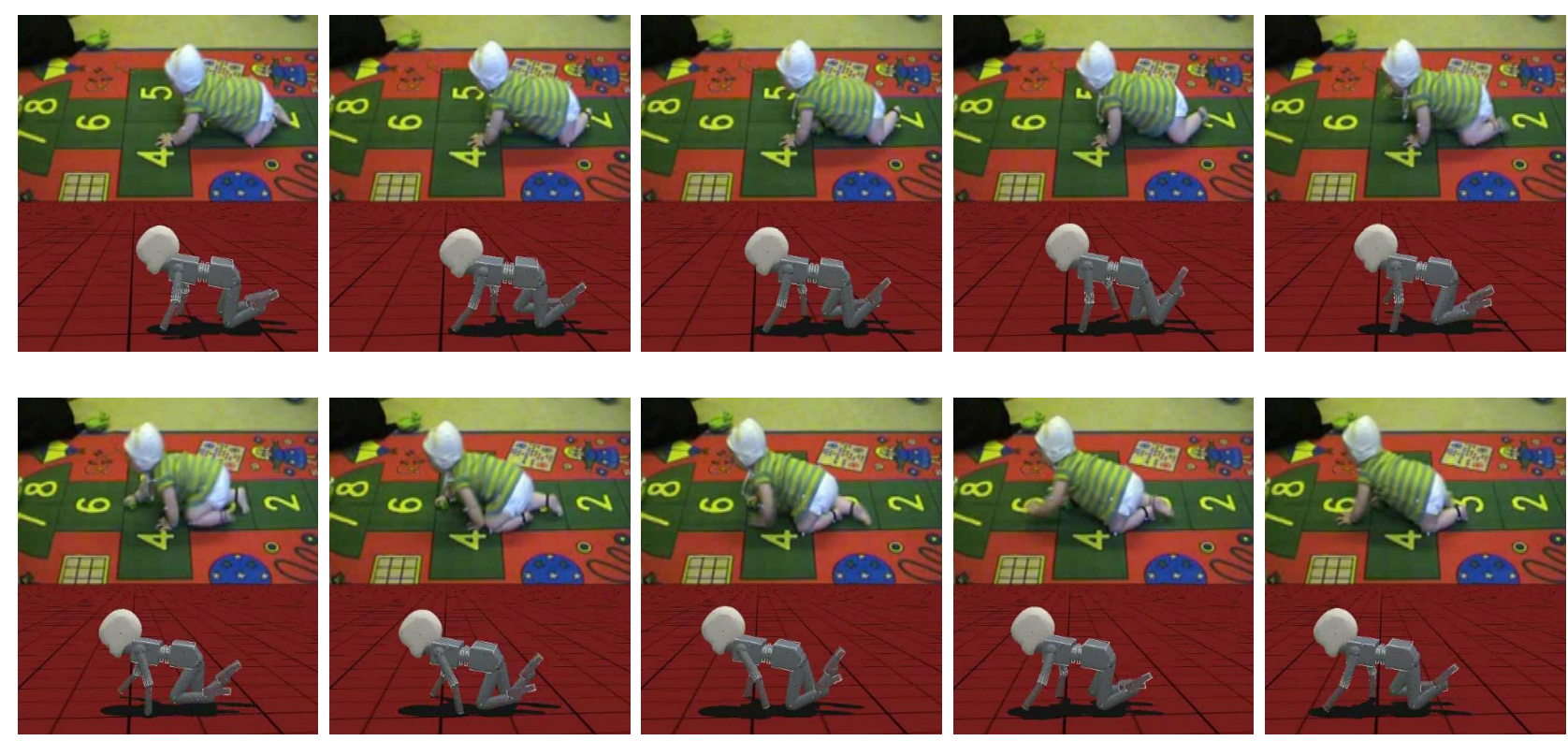

Fig. 10. We show a sequence of crawling of both a real baby and the simulated robot. There is an interval of $120 \mathrm{~ms}$ between each picture.

The result of this experiment can be seen in Figure 9. We see in this figure that the simulated robot could crawl up to 0.3 $\mathrm{m} \cdot \mathrm{s}^{-1}$. A baby crawls around $0.18 \mathrm{~m} \cdot \mathrm{s}^{-1}$ [17], so the robot can attain the speed of a real baby. In this figure, we see that increasing $k_{\text {stance }}$ increases the speed of the robot. However, we see that beyond a certain value the speed of locomotion becomes really small, which corresponds to cases where the robot falls. We also note that increasing $k_{\text {swing }}$ for a given $k_{\text {stance }}$ does not lead to a significantly faster crawling. This seems normal since the swing phase is not the longer part of the movement. However the value of $k_{\text {swing }}$ is important for the stability of locomotion, a too slow swing phase leads to cases where the robot falls or the swinging arm touches the ground too early, in the middle of the swing phase.

\section{CONCLUSion}

In this contribution, we presented an oscillator in which we can independently control the duration of the ascendant and descendant phases. In locomotion control it enables us to set the duration of the swing and the stance phases separately.

We also presented an original way of coupling two oscillators, in order to reproduce the inhibited movement of a limb during the swing phase of the opposite limb. Moreover, we showed that we can use the theory of symmetric coupled cells to construct the architecture of a network of coupled oscillators, given a desired symmetry in the oscillations. This also allowed us to derive the other patterns of oscillations our network could support in a very simple manner, transforming the analytic problem into an algebraic one. The CPG we constructed has several properties that are relevant to robotics: it is stable against perturbations, which is good for sensory feedback integration, and we can easily modulate the pattern in frequency and amplitude.
Finally we showed that our model of CPG matched quite well the experimental data of crawling babies and we showed that it could be successfully used to control a simulated humanoid robot.

The oscillator we constructed and the design methodology we followed to build the network of coupled oscillators are general enough to be used in many other applications where rhythmic pattern generation is necessary.

Our future research goals will be to explore how we can integrate sensory feedback to deal with unexpected perturbations and how we can also control the direction of locomotion with this CPG. Finally we will test this controller on the real robot, as soon as it is built.

\section{ACKNOWLEDGMENT}

We would like to gratefully acknowledge Kerstin Rosander, Ines Halberstadt and Heiko Backes from Uppsala University (Sweden) for providing the kinematic data of crawling babies.

This work was made possible thanks to the support of the European Commission's Cognition Unit, project no. IST2004-004370: RobotCub (L.R.) and to a grant from the Swiss National Science Foundation (A.I.).

\section{REFERENCES}

[1] G. Sandini, G. Metta, and D. Vernon, "Robotcub: an open framework for research in embodied cognition," 2004, paper presented at the IEEE-RAS/RJS International Conference on Humanoid Robotics, Santa Monica, CA.

[2] S. Grillner, "Neurobiological bases of rhythmic motor acts in vertebrates," Science, vol. 228, no. 4696, pp. 143-149, 1985.

[3] G. N. Orlovsky, T. G. Deliagina, and S. Grillner, Neuronal control of locomotion: from mollusc to man. Oxford University Press, 1999.

[4] J. Collins and S. Richmond, "Hard-wired central pattern generators for quadrupedal locomotion," Biological Cybernetics, vol. 71, no. 5, pp. 375-385, 1994.

[5] N. Kopell and G. Ermentrout, "Coupled oscillators and the design of central pattern generators," Mathematical Biosciences, vol. 90, no. 1-2, pp. 87-109, 1988. 
[6] G. Endo, J. Nakanishi, J. Morimoto, and G. Cheng, "Experimental studies of a neural oscillator for biped locomotion with qrio," in Proceedings of the 2005 IEEE International Conference on Robotics and Automation, Barcelona, Spain, 2005, pp. 598-604.

[7] H. Kimura, S. Akiyama, and K. Sakurama, "Realization of dynamic walking and running of the quadruped using neural oscillators," $\mathrm{Au}$ tonomous Robots, vol. 7, no. 3, pp. 247-258, 1999.

[8] G. Taga, "Emergence of bipedal locomotion through entrainment among the neuro-musculo-skeletal system and the environment," Physica D: Nonlinear Phenomena, vol. 75, no. 1-3, pp. 190-208, 1994.

[9] A. Ijspeert, J. Nakanishi, and S. Schaal, "Learning rhythmic movements by demonstration using nonlinear oscillators," in Proceedings of the IEEE/RSJ Int. Conference on Intelligent Robots and Systems (IROS2002), 2002, pp. 958-963.

[10] L. Righetti and A. Ijspeert, "Programmable central pattern generators: an application to biped locomotion," in International Conference on Robotics and Automation (ICRA2006), 2006.

[11] T. A. McMahon, Muscles, Reflexes, and Locomotion. Princeton University Press, 1984.

[12] P. Buono and M. Golubitsky, "Models of central pattern generators for quadruped locomotion. i. primary gaits." Journal of Mathematical Biology, vol. 42, no. 4, pp. 291-326, 2001.

[13] M. Golubitsky and I. Stewart, The symmetry perspective: from equilibrium to chaos in phase space and physical space. Basel, Boston, Berlin: Birkhäuser, 2002.

[14] _ - "Nonlinear dynamics of network: the groupoid formalism," Bulletin of the American Mathematical Society, vol. 43, pp. 305-364, 2006.

[15] K. E. Adolph, B. Vereijken, and M. A. Denny, "Learning to crawl," Child Development, vol. 69, no. 5, pp. 1299-1312, October 1998

[16] E. Goldfi eld, "Transition from rocking to crawling: postural constraints on infant movement," Developmental Psychology, vol. 25, no. 6, pp. 913-919, 1989.

[17] C. Niemitz, "Kinematics and ontogeny of locomotion in monkeys and human babies," Zeitschrift fur Morphologie und Anthropologie, vol. 83, pp. 383-400, March 2002.

[18] A. Pikovsky, R. Rosenblum, and J. Kurths, Synchronization, A universal concept in nonlinear sciences, ser. Cambridge Nonlinear Science Series. Cambridge University Press, Cambridge, UK, 2001, vol. 12.

[19] O. Michel, "Webots: Professional mobile robot simulation," International Journal of Advanced Robotic Systems, vol. 1, no. 1, pp. 39-42, 2004.

[20] Open dynamic engine documentation. [Online]. Available: http://www.ode.org 